\title{
Clinical characteristics of moderate and severe cases with COVID-19 in Wuhan, China: a retrospective study
}

\author{
Lingshuang Sheng ${ }^{1} \cdot$ Xiong Wang $^{2} \cdot$ Ning Tang $^{2} \cdot$ Fankai Meng $^{1} \cdot$ Liang Huang $^{1} \cdot$ Dengju Li $^{1}$
}

Received: 5 May 2020 / Accepted: 11 September 2020 / Published online: 19 September 2020

(c) Springer Nature Switzerland AG 2020

\begin{abstract}
With the outbreak of COVID-19 ongoing, this infectious disease has been posing a significant threat to public health. However, we are still relatively inexperienced on recognizing the clinical characteristics of severe COVID-19 and death cases. Therefore, we hereby collected and analyzed a total of 232 cases to illustrate the clinical characteristics of such patients in Wuhan and to find notable marks for early clinical warning. We consider age, comorbidities, platelet count, albumin, D-dimer, LDH, CRP and IL-6 level might be more meaningful marks for COVID-19 prognostic evaluation.
\end{abstract}

Keywords COVID-19 $\cdot$ SARS-cov-2 Clinical characteristics

\section{Introduction}

Coronavirus disease 2019 (COVID-19) is widely known as a type of severe infectious disease primarily transmitted via respiratory tract and body contact. It has demonstrated a series of clinical characteristics including long incubation period, strong infectivity, high occurrence of multiple organ dysfunction syndrome (MODS) and high mortality rate among severe cases. The infectious disease has been posing a significant threat to public health $[1,2]$. On January 31,2020 , World Health Organization (WHO) officially announced COVID-19 as a public health emergency of international concern (PHEIC). As of July 2020, the COVID-19 pandemic has affected more than 200 countries and regions

Article Summary Line At this time being, COVID-19 is one of the biggest research focus in the world. This manuscript collected a large number of severe and death cases with COVID-19 in Wuhan, China, which could provide more precise and potential indexes for prognostic evaluation and be useful for early warning and subsequent treatment decisions.

Dengju Li

lidengju@163.com

1 Department of Hematology, Tongji Hospital of Tongji Medical College of Huazhong University of Science and Technology, No. 1095 Jie Fang Avenue, Hankou, Wuhan, Hubei, China

2 Clinical Laboratory, Tongji Hospital of Tongji Medical College of Huazhong University of Science and Technology, Wuhan, Hubei, China worldwide, with a total of more than 13 million confirmed cases and over 57,000 deaths [3].

The main clinical manifestations of COVID-19 are fever, dry cough and short of breath. Gastrointestinal symptoms and liver damage could also be observed in some cases $[4,5]$. Severe patients might progress to dyspnea and even acute respiratory distress syndrome (ARDS), immunological derangement, coagulopathy, septic shock and MODS [6]. As a recent report showed that patients with COVID-19 could also develop the symptoms of anosmia and ageusia [7], we should also be alert for new clinical symptoms caused by the latent mutation of COVID-19 virus.

During this study, we collected and analyzed a total of 232 cases from intensive care unit (ICU) and general wards of Wuhan Tongji Hospital. Our aim was to retrospectively analyze the clinical characteristics of these cases and compare the differences between moderate and severe cases and between survived and death cases, respectively, so as to find some early warning clues for future clinical treatment.

\section{Methods}

\section{Data collection}

All data were collected from patients diagnosed with COVID-19 according to the diagnostic criteria issued by National Health Commission of the People's Republic of China [8], who were hospitalized in Wuhan Tongji Hospital 
during the study period. All cases were categorized into two main groups: moderate cases and severe cases (including critical cases). Severe cases met at least one of the following criteria: 1 . respiratory distress with respiratory rate over 30 per minute; 2 . oxygen saturation $\leq 93 \%$ under resting state; 3 . arterial blood oxygen partial pressure $\left(\mathrm{PaO}_{2}\right)$ /oxygen concentration $\left(\mathrm{FiO}_{2}\right) \leq 300 \mathrm{mmHg} ; 4$. chest imaging tests suggesting $>50 \%$ progression of lung lesions within $24-48 \mathrm{~h}$. After analyzing our raw data and data from other COVID19 reports [2, 6, 9-12], we finally picked up markers that worth further analyzing from age, gender, blood cell count, blood biochemical profile, coagulation function and inflammatory factors. Age, leucocytes, neutrophils, lymphocytes, hemoglobin, platelet, total protein, albumin, globulin, lactate dehydrogenase (LDH), C-reactive protein (CRP), procalcitonin (PCT), D-dimer, fibrinogen, interleukin-6 (IL-6) and interleukin-2 receptor (IL-2R) of all subjects were recorded as clinical characteristics for further analysis.

\section{Viral RNA examination}

All patients were confirmed by COVID-19 viral RNA examinations which were conducted by clinical laboratory of Wuhan Tongji hospital. Their nasal and pharyngeal swab specimens were collected for RT-PCR assay. On receipt of the samples, viral RNA extraction was conducted with magnetic viral RNA/DNA extraction kit on PAN9600 Automated Nucleic Acid Extraction System (Tianlong, Xi' an, China), according to the manufacturer's instructions, followed by polymerase-chain-reaction (PCR) screening for the presence of 2019 novel coronavirus (2019-nCoV) with commercial kits (Tianlong, Xi' an, China) in a volume of $25 \mu \mathrm{L}$ PCR mixture containing $17.5 \mu \mathrm{L}$ reaction solution, $1.5 \mu \mathrm{L}$ probes, $1.5 \mu \mathrm{L}$ Taq and $5 \mu \mathrm{L}$ nucleic acid. Conditions for the amplifications include reverse transcription at $50{ }^{\circ} \mathrm{C}$ for $30 \mathrm{~min}$ and pre-denaturation at $95^{\circ} \mathrm{C}$ for $10 \mathrm{~min}$, followed by five cycles of $94{ }^{\circ} \mathrm{C}$ for $15 \mathrm{~s}, 50{ }^{\circ} \mathrm{C}$ for $30 \mathrm{~s}$ and $72{ }^{\circ} \mathrm{C}$ for $30 \mathrm{~s}$ and 40 cycles of $94{ }^{\circ} \mathrm{C}$ for $10 \mathrm{~s}$ and $58^{\circ} \mathrm{C}$ for $30 \mathrm{~s}$ for fluoresce detection. A cycle threshold value $(\mathrm{Ct}$ value) $\leq 37$ was defined as a positive test, which was based on the recommendation by the National Institute for Viral Disease Control and Prevention (China).

\section{Statistical analysis}

Collected data were analyzed with Statistical Package for the Social Sciences (SPSS) version 20.0 software. Results were shown as median and interquartile range (IQR) values. Independent group $t$ tests were used for the comparison of means for continuous variables that were normally distributed; conversely, the Mann-Whitney $U$ test was used for continuous variables not normally distributed. Proportions for categorical variables were compared using the $\chi^{2}$ test.
Two-sided $P$ values of less than 0.05 were considered statistically significant.

\section{Results}

\section{Age and comorbidities}

The 232 COVID-19 patients ranged in age from 20 to 88 years old, with a median age of 68 years old. The median age was 65 years old in the moderate group (102 cases), and the median age was 71 years old in severe group (130 cases). 88 patients died in the severe group, with the median age of 75 years old, which was significantly higher than that of survivors with the median age of 67 years old $(P<0.05)$. Meanwhile, the age of the severe group was significantly higher than that of the moderate group $(P<0.001)$ (Table 1$)$.

Of the 232 patients with COVID-19, 133 (57.3\%) patients had comorbidities (i.e., hypertension, diabetes, chronic pulmonary disease), and patients with comorbidities were more likely to become severe cases. However, we did not find any specific comorbidities that could contribute to disease progression (Table 1).

\section{Blood cell count}

Blood count analysis showed that the levels of leukocytes and neutrophils in the severe group were significantly higher than those in the moderate group (all $P<0.001$ ). Lymphocyte count and platelet decrease were also more significant in the severe group (all $P<0.001$ ). However, there were no significant differences in white blood cells, neutrophils and lymphocytes between the death group and the survivors (all $P>0.05)$. Besides, the platelet count in the death group was significantly lower $(P<0.05)$. In terms of hemoglobin level, there was no significant difference between the severe group and the moderate group and between the survivors and the death group (all $P>0.05$ ). Analysis of fibrinolysis indicators showed that D-dimer $(P<0.001)$ and fibrinogen $(P<0.05)$ in the severe group were significantly higher than those in the moderate group $(P<0.001$ and $P<0.05)$. The difference of D-dimer between the survival group and the death group was significant $(P<0.001)$ instead of the difference in fibrinogen level $(P>0.05)$ (Table 1).

\section{Blood biochemical profiles, coagulation function and inflammatory factors}

Analysis of blood biochemical indicators found that severe group had significantly lower albumin $(P<0.001)$, higher globulin $(P<0.05)$ and higher LDH level $(P<0.001)$ than those of moderate group. Between the survival group and the death group, only albumin (median $P<0.05$ ) and LDH 
Table 1 Clinical characteristics of patients with COVID-19

\begin{tabular}{|c|c|c|c|c|c|}
\hline & \multirow[t]{3}{*}{ Normal range } & \multicolumn{4}{|l|}{ Median (IQR) } \\
\hline & & \multirow[t]{2}{*}{ All cases $(n=232)$} & \multirow[t]{2}{*}{ Moderate cases $(n=102)$} & \multicolumn{2}{|l|}{ Severe cases $(n=130)$} \\
\hline & & & & Survivors $(n=42)$ & Deaths $(n=88)$ \\
\hline Age (median, IQR, range, years) ${ }^{a, b}$ & & 68 (58-90), 20-88 & 65 (52-70), 20-87 & $67(55-81), 35-88$ & $75(68-81), 47-86$ \\
\hline \multicolumn{6}{|l|}{ Comorbidities } \\
\hline Yes $^{\mathrm{a}}$ & & $133(57.3 \%)$ & $36(35.3 \%)$ & $29(69.0 \%)$ & $68(77.3 \%)$ \\
\hline Chronic pulmonary diseases & & $8(3.4 \%)$ & $1(1.0 \%)$ & $2(4.8 \%)$ & $5(5.7 \%)$ \\
\hline Hypertension & & $77(33.2 \%)$ & $32(31.4 \%)$ & $17(40.5 \%)$ & $28(31.8 \%)$ \\
\hline Diabetes & & $25(10.8 \%)$ & $8(7.8 \%)$ & $5(11.9 \%)$ & $12(13.6 \%)$ \\
\hline Cardiovascular diseases & & $25(10.8 \%)$ & $9(8.8 \%)$ & $6(14.3 \%)$ & $10(11.4 \%)$ \\
\hline Chronic liver diseases & & $12(5.2 \%)$ & $5(4.9 \%)$ & $3(7.1 \%)$ & $4(4.5 \%)$ \\
\hline Chronic kidney diseases & & $10(4.3 \%)$ & $4(3.9 \%)$ & $2(4.8 \%)$ & $4(4.5 \%)$ \\
\hline Malignant tumor & & $6(2.6 \%)$ & $2(2.0 \%)$ & $1(2.4 \%)$ & $3(3.4 \%)$ \\
\hline \multicolumn{6}{|l|}{ Laboratory findings } \\
\hline Leucocytes $\left(\times 10^{9} / \mathrm{L}\right)^{\mathrm{a}}$ & $3.5-9.5$ & $6.74(4.82-8.91)$ & $5.40(4.40-7.34)$ & $7.30(5.72-9.99)$ & $8.06(6.05-13.44)$ \\
\hline Neutrophils $\left(\times 10^{9} / \mathrm{L}\right)^{\mathrm{a}}$ & $1.8-6.3$ & $4.53(2.96-7.29)$ & $3.55(2.63-4.78)$ & $5.47(3.95-8.72)$ & $7.19(4.66-11.85)$ \\
\hline Lymphocytes $\left(\times 10^{9} / \mathrm{L}\right)^{\mathrm{a}}$ & $1.1-3.2$ & $0.98(0.62-1.46)$ & $1.34(0.95-1.74)$ & $0.82(0.55-1.12)$ & $0.62(0.47-0.95)$ \\
\hline Hemoglobin (g/L) & $130-175$ & $127(117-138)$ & $126(120-133)$ & $131(116-141)$ & $132(116-141)$ \\
\hline Platelet $\left(\times 10^{9} / \mathrm{L}\right)^{\mathrm{a}, \mathrm{b}}$ & $125-350$ & 214 (149-297) & $246(194-337)$ & $215(172-300)$ & $137(103-214)$ \\
\hline Total protein $(\mathrm{g} / \mathrm{L})$ & $64-83$ & $68.2(64.7-71.6)$ & $68.7(65.4-71.7)$ & $68.5(64.4-74.5)$ & $66.2(63.5-70.7)$ \\
\hline $\operatorname{Albumin}(\mathrm{g} / \mathrm{L})^{\mathrm{a}, \mathrm{b}}$ & $35-52$ & $34.9(31.4-40)$ & $39.1(34.9-42.3)$ & $33.7(31.6-37.5)$ & $31.2(28.8-33.2)$ \\
\hline Globulin $(\mathrm{g} / \mathrm{L})^{\mathrm{a}}$ & $20-35$ & $32.4(28.4-36.8)$ & $29.5(26.5-33)$ & $35.7(31.8-37.5)$ & $36.4(32.8-40)$ \\
\hline Lactate dehydrogenase $(\mathrm{U} / \mathrm{L})^{\mathrm{a}, \mathrm{b}}$ & $135-225$ & $262(200-460)$ & $209(171-265)$ & $310(207-465)$ & $554(370-808)$ \\
\hline C-reactive protein $(\mathrm{mg} / \mathrm{L})^{\mathrm{a}, \mathrm{b}}$ & $<1.0$ & $19.1(2.7-103.7)$ & $3.9(0.7-13.4)$ & $19.9(12.4-117.4)$ & $114.9(64.4-147.7)$ \\
\hline Procalcitonin $(\mathrm{ng} / \mathrm{mL})^{\mathrm{a}}$ & $<0.05$ & $0.10(0.05-0.21)$ & $0.06(0.05-0.08)$ & $0.10(0.08-0.35)$ & $0.28(0.15-0.62)$ \\
\hline D-dimer (ug/mL FEU) $)^{\mathrm{a}, \mathrm{b}}$ & $<0.5$ & $1.30(0.33-4.64)$ & $0.38(0.22-1.34)$ & $2.21(1.16-4.59)$ & $5.38(1.59-21)$ \\
\hline Fibrinogen $(\mathrm{g} / \mathrm{L})^{\mathrm{a}}$ & $2-4$ & $4.54(3.39-5.75)$ & $3.85(2.94-6.28)$ & $5.09(4.55-6.28)$ & $5.14(3.95-6.55)$ \\
\hline Interleukin-6 $(\mathrm{pg} / \mathrm{mL})^{\mathrm{a}, \mathrm{b}}$ & $<7$ & $10.75(1.80-30.49)$ & $2.64(1.50-6.30)$ & $16.71(10.75-28.71)$ & $63.54(23.27-157.40)$ \\
\hline Interleukin-2 receptor $(\mathrm{U} / \mathrm{mL})^{\mathrm{a}}$ & $223-710$ & $644(323-993)$ & $388(259-557)$ & $821(624-1506)$ & $1057(717-1450)$ \\
\hline
\end{tabular}

${ }^{\text {a }}$ Statistically significant index when comparing moderate cases with severe cases

${ }^{\mathrm{b}}$ Statistically significant index when comparing survivors with deaths. $P$ value $<0.05$ is considered to be statistically significant

$(P<0.001)$ were significantly different. Besides, there was no difference at the total protein level between the severe group and the moderate group, nor between the survivors and the dead group (all $P>0.05$ ). Analysis of the included four inflammatory indicators (CRP, PCT, IL-6 and IL-2R) found that there were significant differences in all four indicators between the moderate group and the severe group $(P<0.05)$. Only CRP $(P<0.001)$ and IL-6 $(P<0.05)$ were different between the survival group and the death group (Table 1).

\section{Discussion}

With the outbreak of COVID-19 and its continued spread, a rudimentary understanding of the disease has been gained. However, we are still relatively inexperienced on recognizing the clinical characteristics of severe COVID19 and death cases. Therefore, we summarized the clinical characteristics of 232 COVID-19 patients and analyzed the differences between moderate and severe cases, survivors and deaths. These comparative analyses are useful for early warning and subsequent treatment decisions, with the ultimate goal of improving survival rate in existing critically ill patients.

Our results showed that the mean age of the critically ill patients was older, which was consistent with the findings of other scholars $[9,10]$. The elderly patients have limited organ compensatory function, more basic diseases before infection and more complications during infection. These may be the reasons for the high proportion of severe patients in elderly COVID-19 patients, so more attention should be paid to elderly patients. It has been reported that the COVID-19 was more likely to occur in older men with 
comorbidities [2, 9, 21], which was consistent with our conclusion. However, we did not find any specific comorbidities that could directly contribute to disease progression, indicating that elder patients with chronic comorbidities who have weaker immune functions are more susceptible to COVID19 infection.

Comparison analysis of blood cell count showed that severe cases had higher neutrophils count, lower lymphocytes and platelet count than moderate cases. Besides, death cases had lower platelet count than survival cases. This conclusion largely matches that of previous studies [11, 12], except that decreasing platelet count in death cases should be highlighted, which is possibly related to sepsis complication. Studies on intensive care medicine revealed that platelet count is related to the prognosis of sepsis patients [13, 14], but it is still unclear whether thrombocytopenia is a cause or consequence of sepsis severity and how platelets contribute to sepsis progression. Therefore, we should keep an eye on COVID-19 patients combined with thrombocytopenia and take measures including blood transfusion or anti-inflammatory therapy if necessary. This phenomenon reflects that severe patients experience more intense inflammatory responses which lead to heavier damage of immune cells. Thrombocytopenia might be primarily attributed to consumption, which may be further linked to widespread thrombosis or disseminated intravascular coagulation (DIC). Another reason could be inflammatory response which inhibits the development and maturation of human megakaryocytes in bone marrow.

Fibrinolysis index analysis showed that severe cases had higher fibrinogen and D-dimer level than moderate cases. Besides, death cases had higher D-dimer level than survival cases. This difference suggests that fibrinolysis was inhibited in severe cases of COVID-19. Extensive thrombus formation was also found at autopsy in patients who died from COVID-19 [15]. Published COVID-19-related coagulation studies have also confirmed the prevalence of hypercoagulation [16-18]. All of these phenomena support the necessity of prophylactic anticoagulation in patients without anticoagulant contraindications.

There were also differences in peripheral blood biochemical and inflammatory indicators between severe cases and moderate cases, which are similar to previous recognition about COVID-19 [19, 20]. This is probably related to the degree of difference on liver injury and inflammatory response between them. It is more necessary to actively improve organ function, fight infection and inflammation to severe patients.

With the rapid control of the outbreak in Wuhan, it is not easy to collect more cases for analysis, which limits our research. But given all the above, we consider age, platelet count, albumin, D-dimer, LDH, CRP and IL-6 level might be more meaningful marks for early prognostic evaluation of COVID-19. Our analysis of COVID-19 patients' clinical data showed that age, fibrinolytic factors, degree of inflammation, and degree of liver function damage all significantly affected the prognosis of patients and provided data from the early stage of this pandemic for further systematic research, which is the strength of our study. Through the clinical observation of large number of cases and the autopsy of more dead patients, we will surely obtain more abundant clinical information and formulate more reasonable plans or measures for the control and prevention of the global ravages of COVID-19.

\section{Conclusions}

In summary, we consider age, comorbidities, platelet count, albumin, D-dimer, LDH, CRP and IL-6 level might be more meaningful marks for COVID-19 prognostic evaluation.

Acknowledgments No other authors have disclosures to declare.

\section{Compliance with ethical standards}

Conflict of interest The authors declare that there is no conflict of interest regarding this article.

\section{References}

1. Wu F, Zhao S, Yu B, et al. A new coronavirus associated with human respiratory disease in China. Nature. 2020;579(7798):265-9.

2. Huang C, Wang Y, Li X, et al. Clinical features of patients infected with 2019 novel coronavirus in Wuhan, China. Lancet. 2020;395(10223):497-506.

3. A public health emergency of international concern over the global outbreak of novel coronavirus declared by WHO. https:// www.who.int/zh/news-room/detail/30-01-2020-statement-on-thesecond-meeting-of-the-international-health-regulations-(2005)emergency-committee-regarding-the-outbreak-of-novel-coron avirus-(2019-ncov).

4. Smyk W, Janik MK, Portincasa P, et al. COVID-19: focus on the lungs but do not forget the gastrointestinal tract. Eur J Clin Invest. 2020;50:e13276.

5. Portincasa P, Krawczyk M, Machill A, et al. Hepatic consequences of COVID-19 infection. Lapping or biting? Eur J Intern Med. 2020;77:18-24.

6. Du Y, Tu L, Zhu P, et al. Clinical features of 85 fatal cases of COVID-19 from Wuhan: a retrospective observational study. Am J Respir Crit Care Med. 2020;201:1372-9.

7. Gautier J-F, Ravussin Y. A new symptom of COVID-19: loss of taste and smell. Obesity. 2020;28:848.

8. National Health Commission of the People's Republic of China. Update on the novel coronavirus pneumonia outbreak (Feb 16, 2020). https://www.nhc.gov.cn/xcs/yqtb/202002/18546da875 d74445bb537ab014e7a1c6.shtml.

9. Qin C, Zhou L, Hu Z, et al. Dysregulation of immune response in patients with COVID-19 in Wuhan, China. Clin Infect Dis. 2020;71:762-8. 
10. Zhang G, Zhang J, Wang B, Zhu X, Wang Q, Qiu S. Analysis of clinical characteristics and laboratory findings of 95 cases of 2019 novel coronavirus pneumonia in Wuhan, China: a retrospective analysis. Respir Res. 2020;21(1):1-10.

11. Henry BM, de Oliveira MHS, Benoit S, et al. Hematologic, biochemical and immune biomarker abnormalities associated with severe illness and mortality in coronavirus disease 2019 (COVID19): a meta-analysis. Clin Chem Lab Med. 2020;58:1021-8.

12. Fu L, Wang B, Yuan T, et al. Clinical characteristics of coronavirus disease 2019 (COVID-19) in China: a systematic review and meta-analysis. J Infect. 2020;80:656-65.

13. Assinger A, Schrottmaier WC, Salzmann M, Rayes J. Platelets in sepsis: an update on experimental models and clinical data. Front Immunol. 2019;10:1687.

14. Nicolai L, Gaertner F, Massberg S. Platelets in host defense: experimental and clinical insights. Trends Immunol. 2019;40(10):922-38.

15. Barnes BJ, Adrover JM, Baxter-Stoltzfus A, et al. Targeting potential drivers of COVID-19: neutrophil extracellular traps. J Exp Med. 2020;217:e20200652.

16. Dolhnikoff M, Duarte-Neto AN, de Almeida Monteiro RA, et al. Pathological evidence of pulmonary thrombotic phenomena in severe COVID-19. J Thromb Haemost. 2020;18:1517-9.
17. Klok FA, Kruip MJHA, van der Meer NJM, et al. Incidence of thrombotic complications in critically ill ICU patients with COVID-19. Thromb Res. 2020;191:145-7.

18. Thachil J. The versatile heparin in COVID-19. J Thromb Haemost. 2020;18:1020-2.

19. Yuan J, Zou R, Zeng L, et al. The correlation between viral clearance and biochemical outcomes of 94 COVID-19 infected discharged patients. Inflamm Res. 2020;69:599-606.

20. Li X, Xu S, Yu M, et al. Risk factors for severity and mortality in adult COVID-19 inpatients in Wuhan. J Allergy Clin Immunol. 2020;146:110-8.

21. Chen N, Zhou M, Dong X, et al. Epidemiological and clinical characteristics of 99 cases of 2019 novel coronavirus pneumonia in Wuhan, China: a descriptive study. Lancet. 2020;395:507-13.

Publisher's Note Springer Nature remains neutral with regard to jurisdictional claims in published maps and institutional affiliations. 